\title{
Pattern regulation of DNA hydrogels formed by lateral phase separation of DNA nanostructures on water-in-oil droplet interfaces
}

\author{
Yusuke Sato ${ }^{a, b,{ }^{*}}$, Masahiro Takinoue ${ }^{\mathrm{b},{ }^{*}}$ \\ ${ }^{a}$ Frontier Research Institute for Interdisciplinary Sciences, Tohoku University, Miyagi 980-8579, \\ Japan \\ ${ }^{\mathrm{b}}$ Department of Computer Science, Tokyo Institute of Technology, Kanagawa 226-8502, Japan
}

${ }^{*}$ Yusuke Sato

Aramaki-aza-aoba 6-6-05, Aoba-ku, Sendai, Miyagi 980-8579, Japan,

Phone: +81-22-795-5849

Email: yusuke.sato.c8@tohoku.ac.jp

* Masahiro Takinoue

4259- J2-36 Nagatsuta-cho, Midori-ku, Yokohama, Kanagawa 226-8502, Japan

Phone: +81-45-924-5680

E-mail: takinoue@c.titech.ac.jp (M.T) 


\begin{abstract}
Phase separation is a key phenomenon in artificial cell construction. Recent studies have shown that the liquid-liquid phase separation of designed-DNA nanostructures induces the formation of liquid-like condensates that eventually become hydrogels by lowering the solution temperature. As a compartmental capsule is an essential artificial cell structure, many studies have focused on the lateral phase separation of artificial lipid vesicles. However, controlling phase separation using a molecular design approach remains challenging. Here, we present the lateral liquid-liquid phase separation of DNA nanostructures that leads to the formation of phase-separated capsule-like hydrogels. We designed three types of DNA nanostructures (two orthogonal and a linker nanostructure) that were adsorbed onto an interface of water-in-oil droplets via electrostatic interactions. The phase separation of DNA nanostructures led to the formation of hydrogels of bicontinuous, patch, and mix patterns, due to the immiscibility of liquid-like DNA during the selfassembly process. The frequency of appearance of these patterns was regulated by designing DNA sequences and altering the mixing ratio of the nanostructures. We constructed a phase diagram for the capsule-like DNA hydrogels by investigating pattern formation under various conditions. Our results provide a method for the design and control of phase-separated hydrogel capsules using sequence-designed DNAs. We envision that by incorporating various DNA nanodevices into DNA hydrogel capsules, the capsules will gain molecular sensing, chemicalinformation processing, and mechano-chemical actuating functions, allowing the construction of functional molecular systems.
\end{abstract}




\section{Main Text \\ Introduction}

Phase separation is a physical phenomenon by which a homogeneous phase separates into two (or more) distinct phases, resulting in nonuniform matter distribution. The phase separation of water-soluble molecules in an aqueous solvent is called liquid-liquid phase separation. A typical example is the phase separation of a polyethylene glycol (PEG)/dextran (DEX) mixture, which forms two liquid phases via segregative phase separation (1). The phase separation of a polymer mixture generally occurs under specific polymer concentrations, temperature, salt concentrations, and polymer molecular weight (2). This phenomenon generates an aqueous two-phase system, adopted in various applications, such as biomolecule extraction (3) and patterning (4).

Phase separation of water-soluble molecules, especially biomolecules, has also been adopted for the construction of artificial cells (5). For example, a cell-free transcription/translation machinery was mixed with a PEG/DEX solution and encapsulated into water-in-oil (W/O) droplets (6). In another example, artificial nucleoid-like structures containing transcriptional reaction sets were formed in lipid vesicles (7). Importantly, phase separation plays crucial roles in living cells, such as heterochromatin formation (8), gene expression regulation (9), and membrane-less organelle construction (10). In cells, proteins and/or nucleic acids exhibit phase separation, leading to the formation of liquid or hydrogel-like molecular condensates $(11,12)$. Given the utility and potential functions of phase separation, controlling it by designing biopolymer molecules is an issue to be addressed in artificial cell studies.

The compartmental capsule is one of the most important structures in artificial cells because it is required for the integration of multiple chemical reactions into a molecular system by separating them from the environment $(13,14)$. W/O droplets $(15-17)$ or lipid vesicles $(18-21)$ have been typically adopted for compartmentalization. The compartmental capsules of living cells, i.e., cellular membrane vesicles, have two-dimensionally segregated domain structures (22) related to biological events, such as signal transduction (23) or molecular uptake (24). Lipid vesicles prepared using two (or more) lipid species with different phase transition temperatures or head group charges exhibit phase separation on lipid bilayer membranes (25-27). Studies have reported methods to control the lateral phase separation of the lipid vesicle capsule by changing the temperature (2527), membrane-tension (26), or lipid composition (27). However, designing and regulating the phase separation of the compartmental capsule using a molecular design approach remains challenging.

Sequence-specific DNA interactions can be used to control the phase separation of DNA nanostructures at the molecular level (28-33). Recent studies have demonstrated that sequencedesigned DNA nanostructures exhibit temperature-dependent phase separation and self-assembly into liquid-like droplets (28-32). The DNA nanostructures formed droplets under a specific temperature and the formed droplets became hydrogels when the temperature was lowered (30). Furthermore, two distinct liquid DNA phases were formed on the two types of DNA nanostructures with orthogonal sequence pairs $(29,30)$, allowing the formation of two immiscible DNA liquid droplets and hydrogels. Designing a DNA-based phase separation system is a feasible approach to the design and control of lateral phase separation of compartmental capsules.

We herein report the formation and regulation of phase-separated DNA hydrogels on W/O droplet interfaces. We used a mixture of cationic and non-ionic surfactants for droplet preparation to enable 
DNA nanostructures to adsorb onto the W/O droplet interface. We designed two types of DNA nanostructures (Y-motif and orthogonal Y-motif, hereafter referred to as orthY-motif), whose sequences are orthogonal to each other, with the ability to form different phases in selective and exclusive self-assembly manners. We investigated the pattern formed on the interface by the selfassembly and phase separation of the motifs using a variety of $Y$ - and orth Y-motif mixture ratios. Then, to address the sequence design-based control of phase separation, we designed additional DNA nanostructures that acted as a linker for the $Y$ - and orth $Y$-motifs. Finally, we constructed a phase diagram by investigating how the addition of the linker changed the phase-separation pattern. While this work only focused on the formation of phase-separated hydrogel capsules on a droplet interface, DNA nanotechnology provides various functional DNA devices, capable of computing (34), sensing (35), and actuating (36), that can be incorporated in the hydrogel capsules. Therefore, our results will serve as the means to design and construct functional microcapsules for artificial cell studies. 


\section{Materials and Methods}

Materials: DNAs were purchased from Eurofins Genomics (Tokyo, Japan). Non-modified and FAM-modified DNAs were of oligonucleotide purification cartridge grade, and Alexa 405- and Cy3modified DNAs were of high-performance liquid chromatography purification grade. DNA was stored in ultrapure water (18 megaohms $\mathrm{cm}$ in resistance) at a concentration of $100 \mu \mathrm{M}$ at $-20^{\circ} \mathrm{C}$ until use. DNA concentration was measured using a microvolume spectrometer (DS-11FX, DeNovix, Wilmington, DE, USA). DNA sequences are provided in Table S1. Sorbitan monooleate (Span 80) and oleylamine were purchased from Tokyo Chemical Industry (Tokyo, Japan) and Kanto Chemical (Tokyo, Japan), respectively. Ultrapure water and $\mathrm{Tris}-\mathrm{HCl}(\mathrm{pH} \mathrm{8.0)}$ were purchased from Thermo Fisher Scientific (Waltham, MA, USA). NaCl powder and liquid paraffin were purchased from FUJIFILM Wako Pure Chemical (Osaka, Japan). Silicone-coated glasses (30 $\mathrm{mm}$ by $40 \mathrm{~mm}$ with a thickness of $0.17 \mathrm{~mm}$ ) were purchased from Matsunami Glass (Osaka, Japan).

Preparation of surfactants in oil solution: A liquid paraffin oil solution containing $20 \mathrm{mM}$ Span 80 and the oleylamine oil solution were respectively prepared as follows. First, Span 80 and liquid paraffin were mixed in a test tube. Similarly, oleylamine was mixed with liquid paraffin. These solutions were well vortexed and then, sonicated for $1 \mathrm{~h}$ at $50^{\circ} \mathrm{C}$ using a sonicator bath (CPX1800h$\mathrm{J}$, Branson, Danbury, CT, USA). After the sonication-treatment, the $20 \mathrm{mM}$ Span 80 and oleylamine solutions were mixed at a molar ratio of $1: 3$ to prepare a $10 \mathrm{mM}$ surfactant solution. The mixture was further sonicated using the sonicator bath for $1 \mathrm{~h}$ at $50^{\circ} \mathrm{C}$. The mixed solution was stored under $\mathrm{N}_{2}$ gas-filling and light-shielding until use at $23^{\circ} \mathrm{C}$.

Generation of water-in-oil droplets containing DNA nanostructures and their annealing: DNA strands were mixed in a PCR tube with a buffer containing $20 \mathrm{mM}$ Tris- $\mathrm{HCl}(\mathrm{pH} 8.0)$ and $350 \mathrm{mM}$ $\mathrm{NaCl}$. The DNA concentrations were altered depending on each experiment. Note that the dyemodified DNA strands without the sticky-end were added at a 10\% molar ratio (e.g., $2.5 \mu \mathrm{M}$ for Y1 and $\mathrm{Y}-3,2.25 \mu \mathrm{M}$ for $\mathrm{Y}-2$, and $0.25 \mu \mathrm{M}$ for the fluorescence-modified strands; Table S1). The surfactant mixture in oil and DNA solution were incubated for $2 \mathrm{~min}$ at $95^{\circ} \mathrm{C}$ using a thermal cycler (MiniAmp Thermal Cycler, Thermo Fisher Scientific, Waltham, MA, USA). Two microliter of DNA solution was added to $50 \mu \mathrm{l}$ of the surfactant mixture in a PCR tube and incubated for $1 \mathrm{~min}$ at $95^{\circ} \mathrm{C}$. Then, the tube was well mixed by tapping to generate micrometer-sized water-in-oil droplets. The tubes containing the droplets were again placed on the thermal cycler, and the temperature was lowered from $95^{\circ} \mathrm{C}$ to $25^{\circ} \mathrm{C}$ at a rate of $-1{ }^{\circ} \mathrm{C} / 10 \mathrm{~s}$ (annealing process). The generated droplet radius was $4.7 \pm 1.3 \mu \mathrm{m}$ (mean \pm standard deviation) (Figure $\mathrm{S} 1$ ).

Observation: After annealing, the prepared droplets were placed onto the silicone-coated glass using a micropipette with a tip that was cut-off to make the diameter larger than the droplets. The bottom surfaces or cross-sections of the droplets were visualized using a confocal laser scanning microscope (FV1000, Olympus, Tokyo, Japan) with a 40x objective lens (UPLAPO, Olympus, Tokyo, Japan). Alexa 405, FAM, and Cy3, were imaged using lasers at 405, 473, and $543 \mathrm{~nm}$, respectively. 


\section{Results and Discussion \\ Phase-separated hydrogel formation}

Span 80 and oleylamine were used to prepare W/O droplets (Figure 1a). Span 80 and oleylamine have the same alkyl chain (C18:1, 9-cis), but different head groups. Span 80 is a non-ionic surfactant that was used for stabilizing the generated droplet. Oleylamine is a cationic surfactant that was used to accumulate the DNA nanostructures onto the droplet interface via electrostatic interactions. As the $\mathrm{p} K_{\mathrm{a}}$ value of oleylamine was around 10.0-10.7 $(37,38)$, the amine part in oleylamine was protonated (positively charged) in our buffer condition ( $\mathrm{pH} 8.0)$. Therefore, the DNA nanostructures with negatively charged-phosphate groups, were adsorbed to the droplet interface. By optimizing the mixing ratio of Span 80/oleylamine for DNA adsorption, we determined that DNA nanostructures were well adsorbed at the Span 80/oleylamine ratio of 1/3 (Figure S2).

Two types of DNA nanostructures, $\mathrm{Y}$ - and orth Y-motif, were designed. The motifs were respectively composed of three different single-stranded DNAs (ssDNAs) (Figure 1b) of equal length (nucleotide (nt) number). Both motifs have three sticky-ends with $8 \mathrm{nt}$ palindrome sequences, however, the sticky-end sequences between the two motifs were not complementary (orthogonal sequences). Thus, each motif could interact with other motifs of the same type. Owing to this design, each motif could selectively and exclusively self-assemble into different hydrogels by forming the network structures of the motifs (Figure 1b). The sequences of both motifs were designed to have approximately the same thermodynamic parameters (melting temperature: $T_{\mathrm{m}}$ ) of hybridization in the formation of the motifs and in the sticky-ends (Table S2) (39). Phase-separated gel networks were formed on the interface by adding the two motifs, the buffer, and salts in an aqueous phase for droplet formation, after the annealing process (Figure 1c).

We investigated hydrogel formation on the droplet interface using an equimolar motif concentration (2.5 $\mathrm{MM}$ each) (Figure 2a). For imaging, $\mathrm{Y}$ - and ${ }^{\text {orth }}$-motifs were labeled with FAM and Alexa405, respectively (fluorescence-modified strands were added at a $10 \%$ molar ratio). Microscopic observation revealed that after the annealing process, the DNA nanostructures exhibited lateral phase separation on the droplet interfaces, thereby the droplets were covered with phase-separated DNA hydrogels that showed various patterns (Figure 2b). We classified the patterns into three types: bicontinuous (BC), Y-motif patch (Y-patch) and ${ }^{\text {orth }} Y$-motif patch (orth $Y$ patch) (Figure 2c). In the BC pattern, both the $Y$ - and ${ }^{\text {orth }} \mathrm{Y}$-motifs formed continuous hydrogels. In the Y-patch pattern, Y-motifs formed smaller hydrogels in a continuous orth $Y$-motif hydrogel, and vice versa in ${ }^{\text {orth }} Y$-patch pattern. The appearance frequency of the patterns was analyzed by observing the droplet surfaces. The results showed that BC pattern was over $70 \%$ under the equimolar condition. There were no significant associations between the pattern and the droplet size (Figure S3).

The formation of the phase-separated gel patterns was attributed to the immiscibility of the $Y$ and ${ }^{\text {orth }} Y$-motifs, which was due to their orthogonal sequences. Our earlier study (30) revealed that in a specific temperature range, the $Y$-motifs self-assembled into liquid-like microstructures (named DNA droplets) and two types of DNA droplets composed of $\mathrm{Y}$ - or orth Y-motifs exhibited selective/exclusive fusion behavior. In this study, under a specific annealing temperature range between approximately 63 and $35^{\circ} \mathrm{C}(30)$, the motifs self-assembled into liquid-like structures on the W/O droplet interface. The two types of "DNA liquids" on the interface were immiscible, resulting in their phase separation. Because the DNA liquids become hydrogels following a temperature decrease (30), phase-separated hydrogel patterns can be formed on the interface. Although DNA 
nanostructures without sticky-ends were adsorbed onto the interfaces, the patterns were not observed (Figure S4), clearly demonstrating that the formation of phase-separated hydrogel patterns depended on the orthogonality of the sticky-end sequences.

\section{Pattern regulation by adjusting the nanostructures mixing-ratio}

To explore the possibility of controlling the patterns on the interface, we focused on the mixingratio of the two motifs. We prepared W/O droplets containing the $\mathrm{Y}$ - and ${ }^{\text {orth }} \mathrm{Y}$-motif mixture at a concentration ratio of $1.5 / 3.5$ or $3.5 / 1.5(\mu \mathrm{M} / \mu \mathrm{M})$. The results showed apparent differences in the trends of the pattern formed between the two conditions (Figure 3). At the $Y /$ orth $Y$ ratio of 1.5/3.5, approximately all droplet interfaces were covered with the Y-patch pattern (Figure 3a). In contrast, most of the droplets were covered with the orthY-patch pattern at the Y/orth $Y$ ratio of 3.5/1.5 (Figure $3 b)$. The frequency analysis of the formed patterns showed that the majority were patch patterns; the $Y$-patch pattern or orth Y-patch pattern frequency was over 90 or $75 \%$ at $Y /$ orth $Y=1.5 / 3.5$ or $3.5 / 1.5$, respectively (Figure 3c). These results showed that the type of patterns formed can be regulated by adjusting the mixing-ratio of the two immiscible motifs.

The formation of patch patterns is expected to be determined during the self-assembly process of the motifs. Under an unbalanced motif concentration, a large number of motifs can easily selfassemble into large hydrogels that can almost entirely cover the droplet interface. In contrast, a smaller number of motifs is difficult to assemble or grow to large hydrogels.

Notably, although the thermodynamic parameters of sticky-end hybridization were approximately the same in both motifs, the BC pattern frequency at $Y /{ }^{\text {orth }} Y=3.5 / 1.5$ was higher than that at

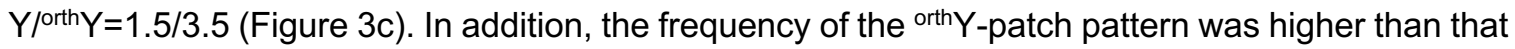
of the Y-patch pattern (Figure 2d), implying that the sticky-end sequence in Y-motifs favors the formation of the continuous hydrogel pattern rather than the formation of patch patterns. Although it is challenging to reveal the detailed mechanisms causing this imbalance, which is out of the scope of the present study, clarifying it would deepen our understanding of the design of DNA nanostructures capable of phase separation.

\section{Pattern regulation by adding sequence-designed linker structures}

In addition to the mixing-ratio of the motifs, the possibility to design a DNA sequence to control the formed patterns was examined. We designed an X-shaped linker (X-linker) with four stickyends, two for the Y-motif and two for the orthY-motif (Figure 4a, left). The X-linkers can form homogeneous hydrogels on the droplet interface (Figure S5), similar to those of either $Y$ - or orthYmotifs (Figure S5). The $\mathrm{X}$-linker is able to crosslink the $\mathrm{Y}$ - and orth $Y$-motifs, resulting in the elimination of orthogonality (Figure $4 a$, right). At a higher $X$-linker concentration, the $Y$ - and ${ }^{\text {orth }} Y$ motifs should be homogeneously distributed on the interface due to cross-bridging. This pattern was named Mix pattern.

The $\mathrm{X}$-linker concentration ranged from 0 to $1 \mu \mathrm{M}$ using a concentration of $2.5 \mu \mathrm{M}$ of $\mathrm{Y}$ - and orthYmotifs. The appearance frequency of the patterns was analyzed using imaging (Figure $4 \mathrm{~b}$ and $\mathrm{c}$ ). At 0 and $0.1 \mu \mathrm{M}$, most of the droplet interfaces were covered with phase-separated hydrogels. At 0.25 and $0.5 \mu \mathrm{M}$, although the Mix pattern formed on some droplets, phase-separated hydrogels were still observed, where the boundary between the hydrogels of each motif was vague and the 
size of patches seemed to become smaller (Figure S6, Supplementally Note 1). At $1 \mu \mathrm{M}$, all droplets were covered with the Mix pattern. To further investigate the effect of the X-linker concentration on Mix pattern appearance, sigmoidal curve fitting of the X-linker concentration-dependent frequency of Mix pattern formation was performed using a Hill-type sigmoidal curve (40) (Figure S7, Supplementally Note 2). The fitting result showed that $0.43 \mu \mathrm{M}$ of the $\mathrm{X}$-linker concentration results in $50 \%$ Mix pattern appearance.

The size decrease of patches (Figure S6) at the X-linker concentrations of 0.25 and $0.5 \mu \mathrm{M}$ was likely attributed to the emulsification of DNA liquid during annealing. Since the $X$-linker linked the

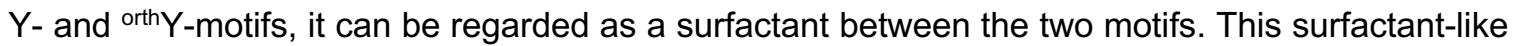
role has been confirmed in previous studies in bulk solution $(29,30)$. Jeon et al., discussed that DNA liquid composed of DNA nanostars can form microemulsions in another DNA liquid using cross-linker nanostars (29). In addition, they discussed that the cross-linkers formed micelles in DNA liquids where the cross-linker nanostars were distributed in the bulk DNA liquid. In the present study, the X-linkers were almost homogeneously distributed in both hydrogels formed by the $\mathrm{Y}$ and ${ }^{\text {orth }} Y$-motifs on the droplet interface (Figure S8), suggesting that the X-linkers also formed micelles in DNA liquid. Thanks to the surfactant-like behavior, the Y-motif (or orthY-motif) DNA liquid in the orthY-motif (or Y-motif) DNA liquid on the interface can be surrounded by the $X$-linkers during annealing, forming DNA liquid emulsions and contributing to forming smaller patches due to the decrease of interfacial tension between the two DNA liquids (29). The emulsified DNA liquids eventually became hydrogels with temperature lowering.

To gain further insight into regulating the patterns of the phase-separated hydrogels, we investigated the effect of $X$-linker concentration on the appearance frequency of the patterns using the $1.5 / 3.5$ or $3.5 / 1.5$ of $Y$-/orth $Y$-motif ratio. The obtained data were fitted using the sigmoidal curve (Figure S7). Based on the results, we constructed a phase diagram of the gel patterns on the interface (Figure 5 and Figure S9). The phase diagram showed that the appearance of the Mix pattern was determined by the amount of the X-linker rather than the Y-/orthY-motif ratio. Notably, using $1 \mu \mathrm{M} X$-linker resulted in the formation of the Mix pattern in all tested $Y$ - and ${ }^{\text {orth }}$-motif concentrations. The phase diagram also suggested that the Mix pattern could appear at lower $X-$ linker concentration under an unbalanced $Y$-/orth $Y$-motif ratio. The appearance of the Mix pattern is a result of DNA liquid emulsification, as discussed above. When the concentration of the one motif is lower than that of the other, X-linker-induced emulsification occurs more easily, leading to high frequency of Mix pattern appearance. Taken together, these findings show that sequence design of the motifs and adjustment of the amount of each motif can be used to regulate the patterns formed on the droplet interface. 


\section{Conclusion}

We constructed phase-separated DNA hydrogels on W/O droplet interfaces, formed by lateral phase separation of two orthogonal DNA nanostructure pairs (Figure 1). Microscopic observation revealed that the two DNA nanostructures self-assembled into phase-separated hydrogels with BC, Y-patch, and orthy-patch patterns (Figure 2). Differences in the mixing-ratio of $Y$ - and ${ }^{\text {orth }} \mathrm{Y}$-motifs changed the appearance frequency of the patterns (Figure 3). Moreover, the addition of the $X$ linkers, which were designed to crosslink the $\mathrm{Y}$ - and ${ }^{\text {orth }} \mathrm{Y}$-motifs, resulted in the formation of the Mix pattern (Figure 4), where both motifs were homogeneously distributed on the interface due to the elimination of orthogonality. Finally, we demonstrated that the patterns of the hydrogels formed by phase separation could be regulated by altering the mixing-ratio of $Y$ - and orth $Y$-motifs and the added amount of the sequence-designed X-linkers, represented as a phase diagram (Figure 5). These results offer an approach to fabricate and control the capsule made of phase-separated hydrogel using sequence-designed DNAs.

Although W/O droplets were widely used as a model for artificial cells, a lipid vesicle is a closer model because the cellular membrane is composed of a lipid bilayer. Our phase-separated hydrogels were formed on a W/O droplet interface. However, we found that hydrogels can also be formed on the droplet surrounded by the cationic and zwitterionic lipid mixture (Figure S10, Supplementally Note 3). Since lipid-surrounded W/O droplets were used as precursors of lipid vesicles in a droplet transfer method (41), lipid vesicles supported by the phase-separated hydrogels can be constructed. This concept was previously demonstrated by Kurokawa et. al. as a DNA-gel cytoskeleton for lipid vesicles (42). In addition, lipid bilayers can phase separate into a cholesterol-rich liquid-ordered phase and cholesterol-less liquid-disordered phase. Because DNA can be modified with cholesterol, controlling the phase separation of lipid bilayers by the phaseseparated hydrogels on the inner leaflet can be expected. Moreover, the lipid-supported hydrogels could be extracted into an aqueous phase by removing the surrounding lipids (43).

Further investigation of the effect of DNA sequences on phase separation may provide a way for precise pattern control. The pattern regulation we showed here did not lead to the formation of targeted patterns, such as an only BC pattern, Y-patch pattern, or orthY-patch pattern. As we discussed in the results shown in Figure $2 \mathrm{~d}$ and Figure $3 \mathrm{c}$, the sticky-end sequences may influence pattern formation. Thus, understanding the relations between the sequence and phase-separated hydrogel formation may lead to the formation of the desired pattern.

We envision the construction of functional microcapsules using our phase-separated hydrogels. The liquid-like state of the assembled DNA nanostructures was based on unstable hybridization of sticky-ends (30), suggesting that DNA hydrogels can attain a liquid-like state by weakening the sticky-end interactions. Photo- (44) or molecular-stimulation (45) can alter the interaction between DNA molecules. Thus, although DNA hydrogels are not dynamic structures, reorganization of the formed patterns could be achieved in response to stimulation. Such stimuli-responsivity may also allow the degradation of targeted pathes in the hydrogels, which offers a cargo release function encapsulated in the spherical hydrogel. Moreover, DNA can be modified with various functional proteins (46), which may enable us to construct phase-separated hydrogel capsules comparable to cellular membranes. Furthermore, DNA molecular devices capable of signal processing (47) or stimuli-responsive actuation $(48,49)$ will be implemented into our micro-sized DNA-hydrogel capsule. We believe that such functional capsules composed of DNA will provide a new approach to developing capsule structures for artificial cell studies and molecular robotics (50). 


\section{Acknowledgments}

We thank Mr. Yuji Nakashima and Ms. Yukiko Okuda for their efforts in preliminary experiments. We also thank Dr. Shin-ichiro M. Nomura for assistance with confocal microscope observations. This work was supported by MEXT/JSPS KAKENHI (grant numbers JP19KK0261, JP20K19918, and JP20H05970 to Y.S., and JP2OH00619, JP2OH05701, JP2OK21828, and JP2OH05935 to M.T.), the NICA Fellows program to Y.S., Research Encouragement Grants from The Asahi Glass Foundation to M.T., and Support for Tokyo Tech Advanced Researchers (STAR) to M.T.

\section{References}

1. H.-O. Johansson, G. Karlström, F. Tjerneld, C. A. Haynes, Driving forces for phase separation and partitioning in aqueous two-phase systems. J. Chromatogr. B: Biomed. Appl. 711, 3-17 (1998).

2. M. Iqbal et al., Aqueous two-phase system (ATPS): an overview and advances in its applications. Biol. Proced. Online 18, 18 (2016).

3. J. Kim, H. Shin, J. Kim, J. Kim, J. Park, Isolation of High-Purity Extracellular Vesicles by Extracting Proteins Using Aqueous Two-Phase System. PLoS One 10, e0129760 (2015).

4. T. I. Farhana et al., Spatial Patterning of Kinesin-1 and Dynein Motor Proteins in an In Vitro Assay using Aqueous Two-Phase Systems (ATPS). Langmuir 35, 13003-13010 (2019).

5. C. D. Crowe, C. D. Keating, Liquid-liquid phase separation in artificial cells. Interface Focus 8, 20180032 (2018).

6. P. Torre, C. D. Keating, S. S. Mansy, Multiphase water-in-oil emulsion droplets for cell-free transcription-translation. Langmuir 30, 5695-5699 (2014).

7. N. N. Deng, W. T. S. Huck, Microfluidic Formation of Monodisperse Coacervate Organelles in Liposomes. Angew. Chem. Int. Ed. Engl. 56, 9736-9740 (2017).

8. A. R. Strom et al., Phase separation drives heterochromatin domain formation. Nature 547, 241-245 (2017).

9. B. R. Sabari et al., Coactivator condensation at super-enhancers links phase separation and gene control. Science 361 (2018).

10. S. Alberti, Phase separation in biology. Curr. Biol. 27, R1097-R1102 (2017).

11. A. Patel et al., A Liquid-to-Solid Phase Transition of the ALS Protein FUS Accelerated by Disease Mutation. Cell 162, 1066-1077 (2015).

12. A. Jain, R. D. Vale, RNA phase transitions in repeat expansion disorders. Nature 546, 243247 (2017).

13. Y. Sato, M. Takinoue, Creation of Artificial Cell-Like Structures Promoted by Microfluidics Technologies. Micromachines 10, 216 (2019).

14. I. Santiago, F. C. Simmel, Self-Propulsion Strategies for Artificial Cell-Like Compartments. Nanomaterials 9, 1680 (2019). 
15. A. Yoshida, S. Kohyama, K. Fujiwara, S. Nishikawa, N. Doi, Regulation of spatiotemporal patterning in artificial cells by a defined protein expression system. Chem. Sci. 10, 1106411072 (2019).

16. L. Aufinger, F. C. Simmel, Artificial Gel-Based Organelles for Spatial Organization of CellFree Gene Expression Reactions. Angew. Chem. Int. Ed. Engl. 57, 17245-17248 (2018).

17. D. Ishikawa et al., DNA Origami Nanoplate-Based Emulsion with Nanopore Function. Angew. Chem. Int. Ed. Engl. 58, 15299-15303 (2019).

18. S. Berhanu, T. Ueda, Y. Kuruma, Artificial photosynthetic cell producing energy for protein synthesis. Nat. Commun. 10, 1325 (2019).

19. S. S. Mansy et al., Template-directed synthesis of a genetic polymer in a model protocell. Nature 454, 122-125 (2008).

20. V. Noireaux, A. Libchaber, A vesicle bioreactor as a step toward an artificial cell assembly. Proc. Natl. Acad. Sci. U. S. A. 101, 17669-17674 (2004).

21. Y. Sato, K. Komiya, I. Kawamata, S. Murata, S. M. Nomura, Isothermal amplification of specific DNA molecules inside giant unilamellar vesicles. Chem. Commun. 55, 9084-9087 (2019).

22. F. R. Maxfield, Plasma membrane microdomains. Curr. Opin. Cell Biol. 14, 483-487 (2002).

23. K. Simons, D. Toomre, Lipid rafts and signal transduction. Nat. Rev. Mol. Cell Biol. 1, 31-39 (2000).

24. L. A. Mulcahy, R. C. Pink, D. R. Carter, Routes and mechanisms of extracellular vesicle uptake. J Extracell Vesicles 3, 24641 (2014).

25. K. Karamdad et al., Engineering thermoresponsive phase separated vesicles formed via emulsion phase transfer as a content-release platform. Chem. Sci. 9, 4851-4858 (2018).

26. T. Hamada, Y. Kishimoto, T. Nagasaki, M. Takagi, Lateral phase separation in tense membranes. Soft Matter 7, 9061-9068 (2011).

27. H. Himeno et al., Charge-induced phase separation in lipid membranes. Soft Matter 10, 7959-7967 (2014).

28. J. Deng, A. Walther, Programmable ATP-Fueled DNA Coacervates by Transient LiquidLiquid Phase Separation. Chem 6, 3329-3343 (2020).

29. B. J. Jeon, D. T. Nguyen, O. A. Saleh, Sequence-Controlled Adhesion and Microemulsification in a Two-Phase System of DNA Liquid Droplets. J. Phys. Chem. B 124, 8888-8895 (2020).

30. Y. Sato, T. Sakamoto, M. Takinoue, Sequence-based engineering of dynamic functions of micrometer-sized DNA droplets. Sci. Adv. 6, eaba3471 (2020). 
31. B. J. Jeon et al., Salt-dependent properties of a coacervate-like, self-assembled DNA liquid. Soft Matter 14, 7009-7015 (2018).

32. D. T. Nguyen, B. J. Jeon, G. R. Abraham, O. A. Saleh, Length-Dependence and Spatial Structure of DNA Partitioning into a DNA Liquid. Langmuir 35, 14849-14854 (2019).

33. S. Biffi et al., Phase behavior and critical activated dynamics of limited-valence DNA nanostars. Proc. Natl. Acad. Sci. U. S. A. 110, 15633-15637 (2013).

34. J. Yin et al., DNA Nanotechnology-based Biocomputing. Chem. Res. Chin. Univ. 36, 219226 (2020).

35. I. Willner, B. Shlyahovsky, M. Zayats, B. Willner, DNAzymes for sensing, nanobiotechnology and logic gate applications. Chem. Soc. Rev. 37, 1153-1165 (2008).

36. M. DeLuca, Z. Shi, C. E. Castro, G. Arya, Dynamic DNA nanotechnology: toward functional nanoscale devices. Nanoscale Horiz. 5, 182-201 (2020).

37. M.-S. Kuo et al., Efficient Dispersants for TiO2 Nanopowder in Organic Suspensions. J. Am. Ceram. Soc. 99, 445-451 (2016).

38. M. Z. Fahmi, J.-Y. Chang, Potential Application of Oleylamine-encapsulated AgInS2-ZnS Quantum Dots for Cancer Cell Labeling. Procedia Chem. 18, 112-121 (2016).

39. N. R. Markham, M. Zuker, DINAMelt web server for nucleic acid melting prediction. Nucleic Acids Res. 33, W577-581 (2005).

40. A. V. HILL, The possible effects of the aggregation of the molecules of haemoglobin on its dissociation curves. J. Physiol. 40, iv-vii (1910).

41. S. Pautot, B. J. Frisken, D. A. Weitz, Production of Unilamellar Vesicles Using an Inverted Emulsion. Langmuir 19, 2870-2879 (2003).

42. C. Kurokawa et al., DNA cytoskeleton for stabilizing artificial cells. Proc. Natl. Acad. Sci. U. S. A. $114,7228-7233(2017)$.

43. M. Weiss et al., Sequential bottom-up assembly of mechanically stabilized synthetic cells by microfluidics. Nat. Mater. 17, 89-96 (2018).

44. H. Asanuma, T. Ito, T. Yoshida, X. Liang, M. Komiyama, Photoregulation of the Formation and Dissociation of a DNA Duplex by Using thecis-trans Isomerization of Azobenzene. Angew. Chem. Int. Ed. Engl. 38, 2393-2395 (1999).

45. S. Okumura et al., Morphological Manipulation of DNA Gel Microbeads with Biomolecular Stimuli. Nanomaterials 11, 293 (2021).

46. O. I. Wilner et al., Enzyme cascades activated on topologically programmed DNA scaffolds. Nat. Nanotechnol. 4, 249-254 (2009).

47. G. Chatterjee, N. Dalchau, R. A. Muscat, A. Phillips, G. Seelig, A spatially localized architecture for fast and modular DNA computing. Nat. Nanotechnol. 12, 920-927 (2017). 
48. Y. Kamiya, H. Asanuma, Light-driven DNA nanomachine with a photoresponsive molecular engine. Acc. Chem. Res. 47, 1663-1672 (2014).

49. J. S. Kahn, Y. Hu, I. Willner, Stimuli-Responsive DNA-Based Hydrogels: From Basic Principles to Applications. Acc. Chem. Res. 50, 680-690 (2017).

50. M. Hagiya, A. Konagaya, S. Kobayashi, H. Saito, S. Murata, Molecular robots with sensors and intelligence. Acc. Chem. Res. 47, 1681-1690 (2014). 
Figures and Tables

(a)

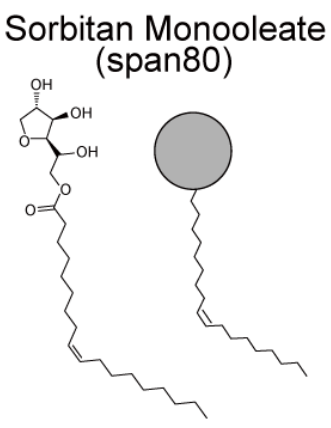

(c)
water-in-oil droplet
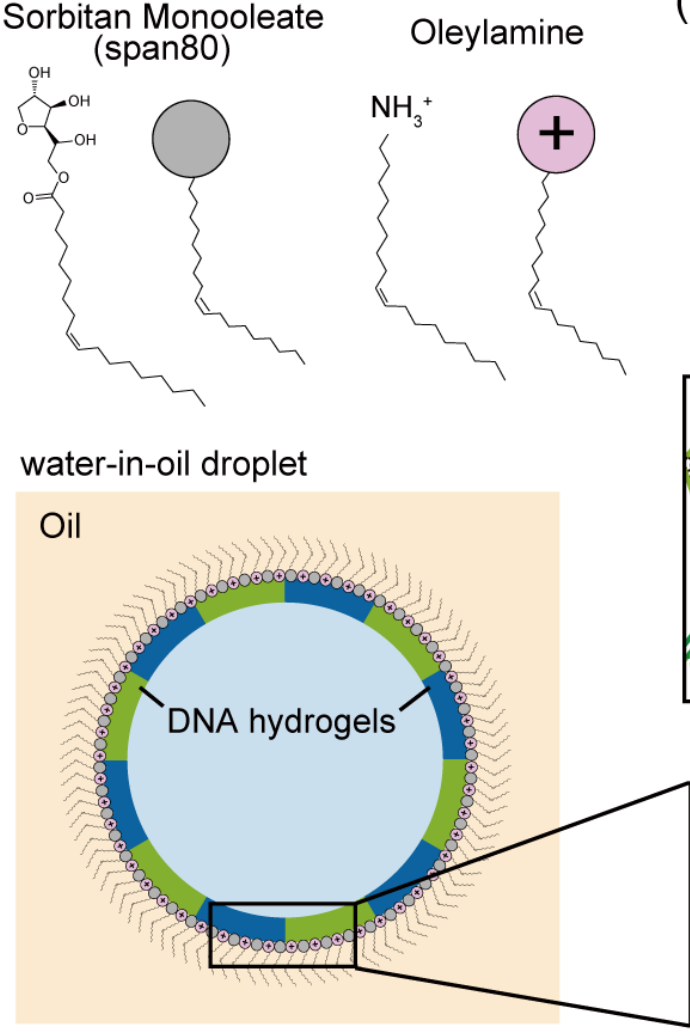

(b)
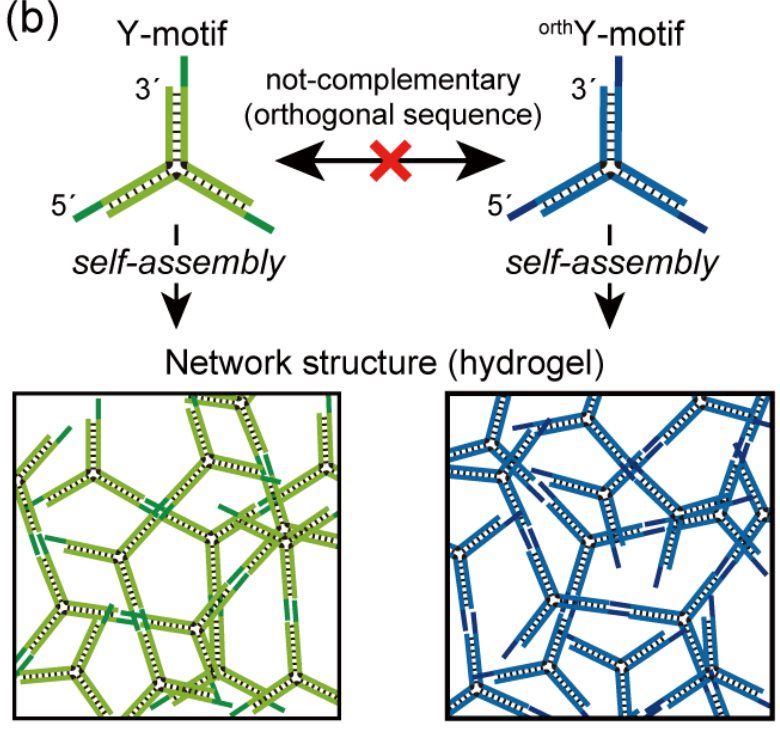

Phase-separated hydrogel

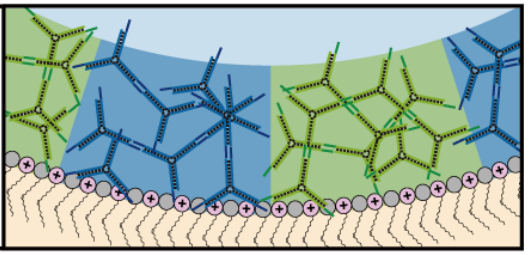

Electrostatic adsorption

Figure 1. Schematic illustrations of the experimental system. (a) Structural formulae of Span 80 and oleylamine. Oleylamine acts as a cationic surfactant due to the protonation of the amine group. (b) DNA nanostructures. The Y-motif and orthogonal Y-motif (orthY-motif) self-assemble into two types of network structures, respectively, as the sticky-ends in the two motifs are orthogonal sequences. (c) Formation of phase-separated DNA hydrogels on a water-in-oil droplet interface. The motifs were adsorbed on the interface via electrostatic interaction, and self-assembled on the interface. 
(a)

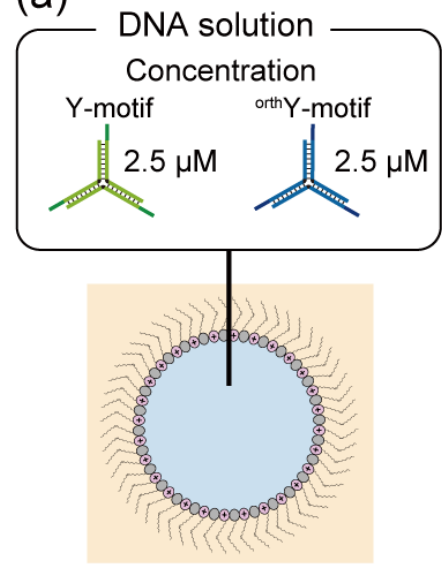

(c)

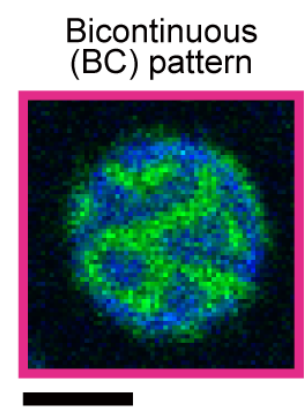

(b)
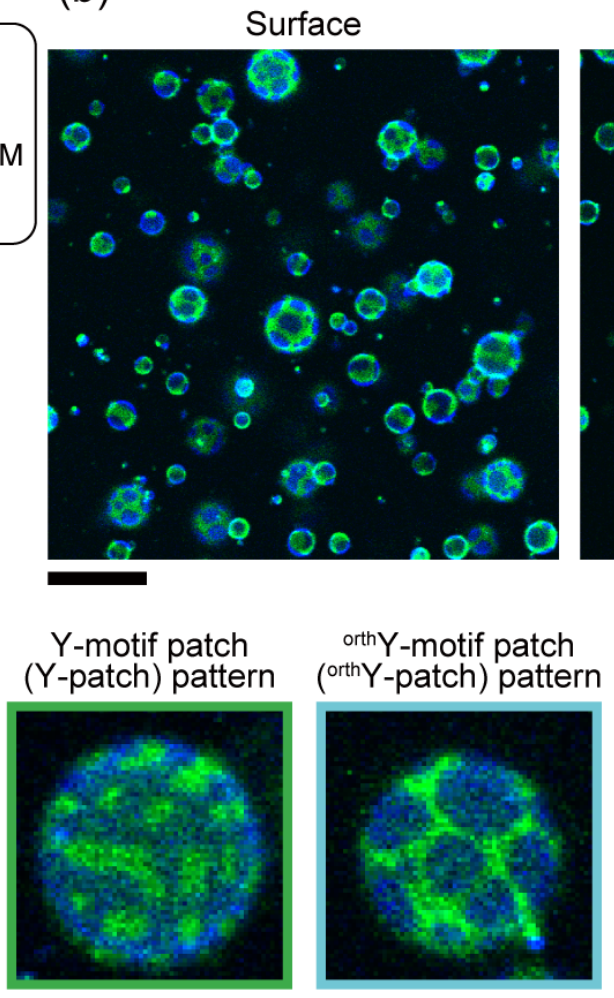

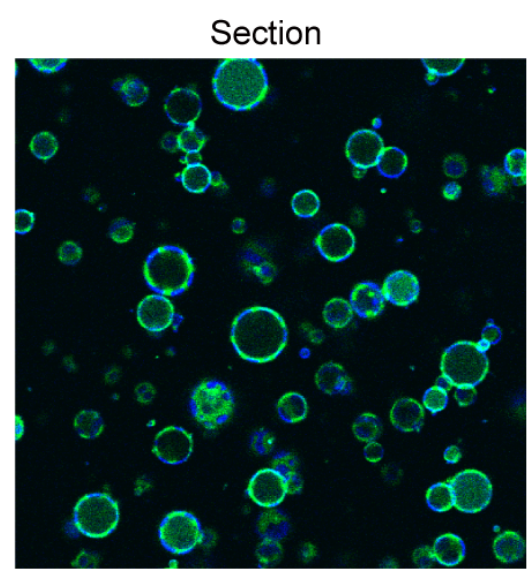

(d)

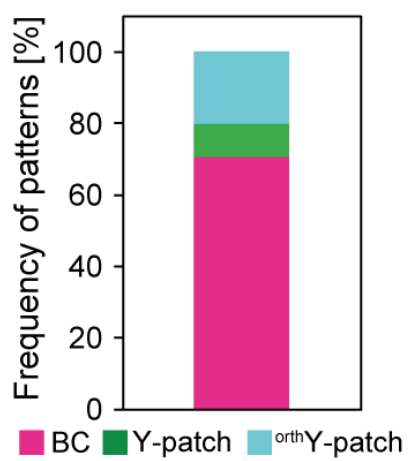

Figure 2. DNA hydrogel patterns on the droplet interface formed by phase separation of two DNA motifs. (a) Schematic representation of the experimental condition. (b) Microscopy images of the droplet surfaces (left) and droplet cross-section (right). The green and blue channels represent the FAM signal for the Y-motif and Alexa405 signal for the orth Y-motif, respectively. (c) Classification of

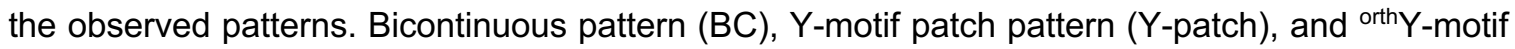
patch pattern (orthY-patch). (d) Pattern frequency. The number of analyzed droplets was 491 in the different experiments. Scale bars: $30 \mu \mathrm{m}$ in (b) and $10 \mu \mathrm{m}$ in (c). 


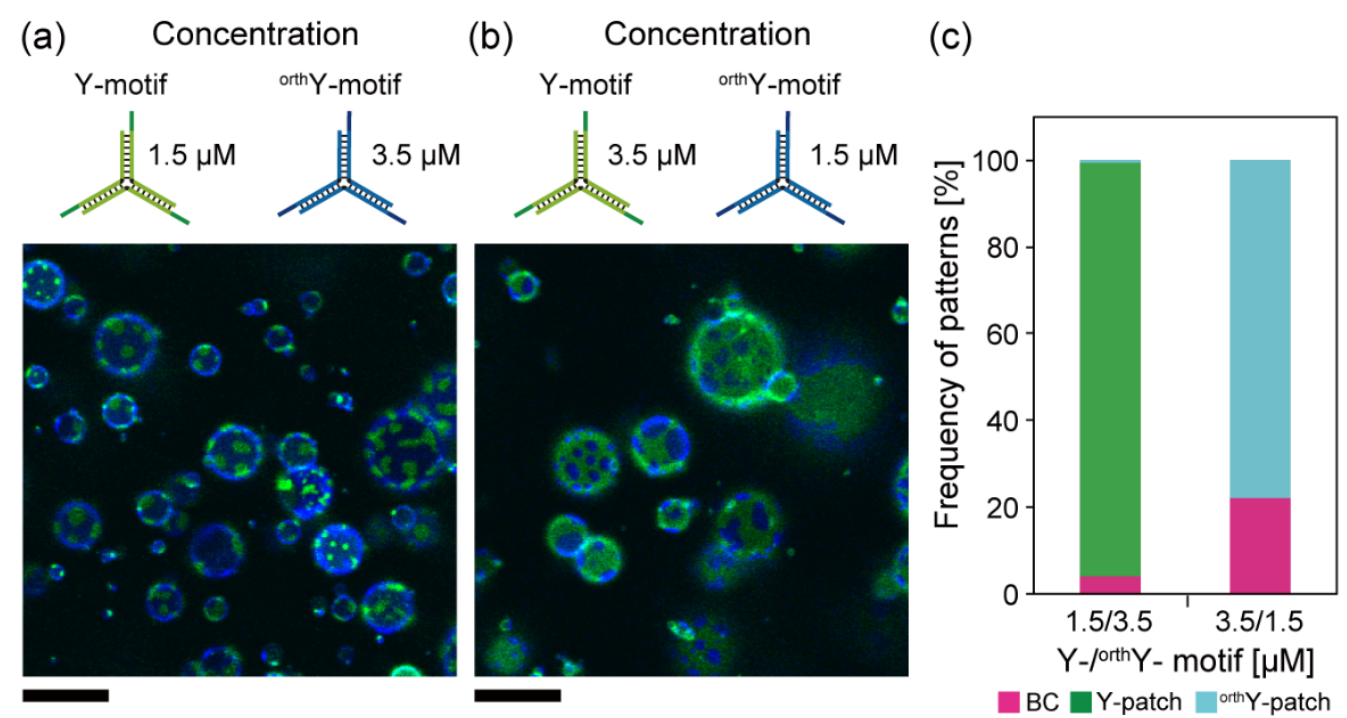

Figure 3. DNA hydrogel patterns on the droplet interface under unbalanced motif concentrations. ( $a$ and $b$ ) Representative microscopy images of the droplet surfaces containing 1.5 and $3.5 \mu \mathrm{M}(\mathrm{a})$ or 3.5 and $1.5 \mu \mathrm{M}(\mathrm{b})$ of the $\mathrm{Y}$ - and orth $Y$-motifs. (c) Frequency of patterns using 1.5/3.5 or 3.5/1.5 $(\mu \mathrm{M} / \mu \mathrm{M})$ of $\mathrm{Y}-$ /orth $\mathrm{Y}$-motifs. The number of analyzed droplets in each condition were 493 for $1.5 / 3.5$ and 343 for $3.5 / 1.5$, respectively. Scale bars: $20 \mu \mathrm{m}$. 
(a)

X-shaped linker
(X-linker)

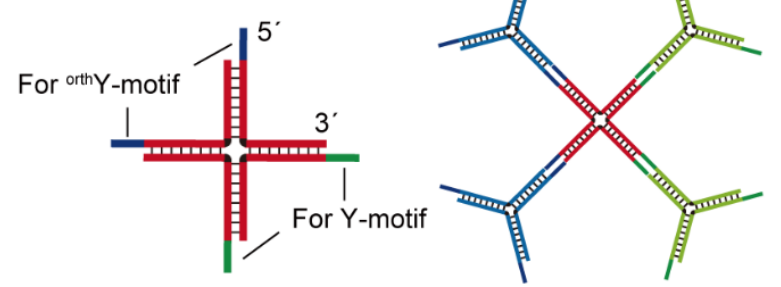

(b)

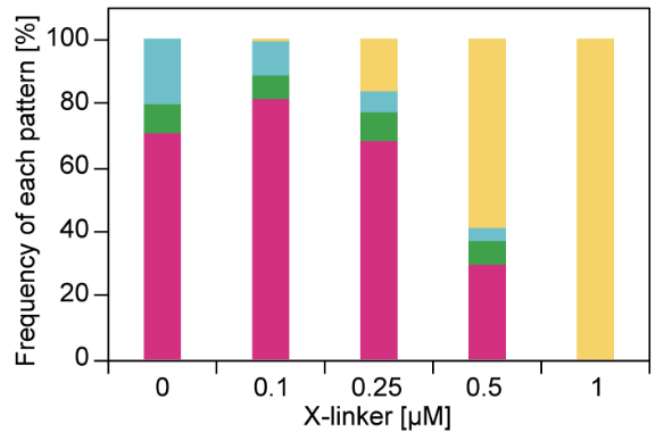

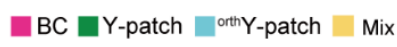

(c)

X-linker $=0 \mu \mathrm{M}$

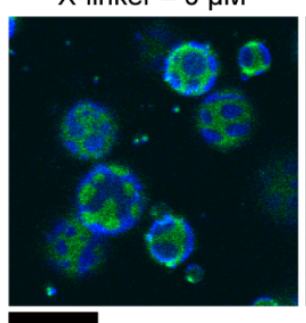

X-linker $=0.1 \mu \mathrm{M}$

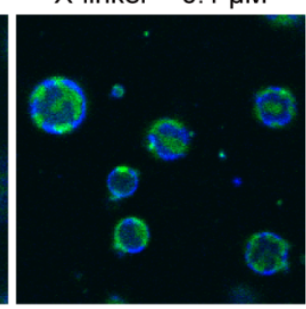

X-linker $=0.25 \mu \mathrm{M}$

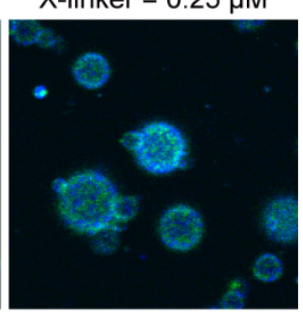

X-linker $=1 \mu \mathrm{M}$
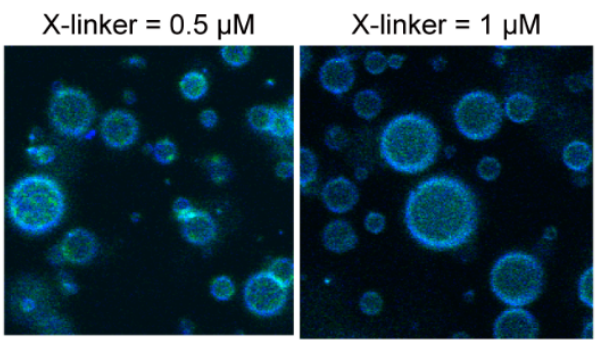

Figure 4. Pattern regulation by the addition of sequence-designed $X$-shaped linker (X-linker). (a) Schematic representation of the X-linker capable of crosslinking the $Y$ - and orth $Y$-motifs. (b) Representative microscopy images of the droplet surfaces containing $2.5 \mu \mathrm{M} Y$ - and ${ }^{\text {orth }} Y$-motif with the designated concentration of the X-linker. Scale bar: $20 \mu \mathrm{m}$. (c) Frequency of patterns under various $\mathrm{X}$-linker amounts. 


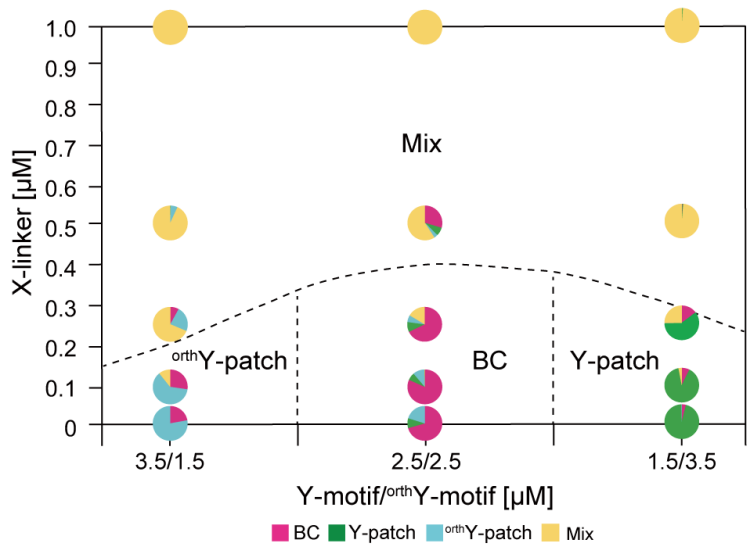

Figure 5. Phase diagram of the hydrogel patterns on the water-in-oil W/O droplet interfaces, representing the effects of the $\mathrm{Y}$ - and ${ }^{\text {orth }} \mathrm{Y}$-motif mixing ratio and the added amount of $\mathrm{X}$-linker on the patterns formed. Note that the dashed lines are eye-guide sketches representing the phase boundary. Pie charts in the phase diagram show the frequency of each pattern. 


\title{
Supplementary Information for
}

Pattern regulation of DNA hydrogels formed by lateral phase separation of DNA nanostructures on water-in-oil droplet interfaces

\author{
Yusuke Sato ${ }^{a, b,{ }^{*}}$, Masahiro Takinoue ${ }^{\mathrm{b},{ }^{*}}$ \\ a Frontier Research Institute for Interdisciplinary Sciences, Tohoku University, Miyagi 980-8579, \\ Japan \\ b Department of Computer Science, Tokyo Institute of Technology, Kanagawa 226-8502, Japan
}




\section{Supplementary Information Text \\ Supplementary Note 1: Method used to analyze the patch size in the phase- separated hydrogels}

The patch size in the phase-separated hydrogels became smaller with the increase in the amount of X-linkers added (Figure $4 \mathrm{~b}$ in the main text). To quantitatively evaluate it, we performed Fourier transform to the fluorescence intensity profiles of the ${ }^{\text {orth }} Y$-motif region on the interface. The intensity line profiles were obtained using the image analysis software ImageJ. We arbitrary drew the lines to the images that visualized the droplet interfaces. The line length was shorter than the droplet because the intensity gap at the oil/water boundary influenced the result. The obtained intensity was standardized so that the average intensity value becomes 0 and dispersion becomes 1 . The standardized data were analyzed using the Fast Fourier Transformation (FFT) with Hanning window function using the Origin software (OriginLab Corp. v 9.60). Twenty droplets were analyzed in each condition. Maximum values in each analysis were used to obtain the mean value, shown in Figure S6.

\section{Supplementary Note 2: Sigmoidal curve fitting of Mix pattern appearance frequency}

We used the following equation for sigmoidal curve fitting:

$$
\text { Mix pattern }[\%]=100 \times[\mathrm{X}-\text { linker }]^{n} /\left(K_{d}+[\mathrm{X}-\text { linker }]^{n}\right)
$$

where $K_{d}$ is the apparent dissociation constant and $n$ is the Hill coefficient (1). $K_{d}$ and $n$ values were determined using a latest square method. The fitting results are shown in Figure S7.

\section{Supplementary Note 3: Preparation of phase-separated hydrogels in lipid- surrounded water-in-oil droplets}

1,2-dioleoyl-sn-glycero-3-phosphocholine (DOPC), a zwitterionic lipid, and 1,2-dioleoyl-3trimethylammonium-propane (DOTAP), a cationic lipid, were employed as an alternative to Span 80 and oleylamine. DOPC and DMTAP were obtained from Avanti Polar lipids 
(Alabaster, USA). The lipid powders were dissolved in a mixture of chloroform/methanol at a 2:1 volume ratio. Each lipid solution was added to round-bottom glass tubes. The organic solvent was evaporated under a nitrogen gas flow and vacuumed to completely remove organic solvents, followed by the addition of liquid paraffin to the glass tube to obtain a $2 \mathrm{mM}$ lipid concentration. The liquid paraffin containing the lipid was sonicated for over $1 \mathrm{~h}$ at $50{ }^{\circ} \mathrm{C}$, and then, equal volumes of the lipid solutions were mixed to obtain a total $2 \mathrm{mM}$ lipid solution (1 $\mathrm{mM}$ each). By using this lipid mixture, droplets containing DNA nanostructures were generated using the same method used with the Span 80/oleylamine mixture. 
Table S1: Oligonucleotide sequences

\begin{tabular}{cc} 
Name & Sequences (5'-3') \\
\hline Y-1 & GCTCGAGCCAGTGAGGACGGAAGTTTGTCGTAGCATCGCACC \\
Y-2 & GCTCGAGCCAACCACGCCTGTCCATTACTTCCGTCCTCACTG \\
Y-3 & GCTCGAGCGGTGCGATGCTACGACTTTGGACAGGCGTGGTTG \\
\hline${ }^{\text {orthy-1 }}$ & CTCGCGAGAAAGGAACTCTCCGCGTTGACAAAGCCGACACGT \\
orthY-2 & CTCGCGAGGCCTCTGTGTCGCATCTTCGCGGAGAGTTCCTTT \\
orthY-3 & CTCGCGAGACGTGTCGGCTTTGTCTTGATGCGACACAGAGGC \\
\hline X-1 & CTCGCGAGGCTGGACTAACGGAACGGTTAGTCAGGTATGCCAGCAC \\
X-2 & GCTCGAGCGTGCTGGCATACCTGACTTTCGCAAATTTACAGCGCCG \\
X-3 & GCTCGAGCCGGCGCTGTAAATTTGCGTTCATCACTTGGGACCATGG \\
X-4 & CTCGCGAGCCATGGTCCCAAGTGATGTTCCGTTCCGTTAGTCCAGC \\
\hline Y-2_0_FAM & [6-FAM] -CAACCACGCCTGTCCATTACTTCCGTCCTCACTG \\
orthY-2_0_Alexa405 & [Alexa405] -GCCTCTGTGTCGCATCTTCGCGGAGAGTTCCTTT \\
X-2_0_Cy3 & [Cy3]-GTGCTGGCATACCTGACTTTCGCAAATTTACAGCGCCG
\end{tabular}


Table S2. Melting temperature $\left(T_{\mathrm{m}}\right)$ of the sequences for the $\mathrm{Y}$ - and ${ }^{\text {orth }} \mathrm{Y}$-motifs. These parameters were obtained using DINAMelt (2) (Two State Melting Hybridization mode) with $350 \mathrm{mM} \mathrm{Na}^{+}$and $2.5 \mu \mathrm{M}$ DNA (for the nanostructures) and $7.5 \mu \mathrm{M} \mathrm{DNA}$ (for the sticky ends)

\begin{tabular}{|c|c|c|}
\hline \multicolumn{2}{|c|}{ Sequence pairs } & $\boldsymbol{T}_{\boldsymbol{m}}\left[{ }^{\circ} \mathrm{C}\right]$ \\
\hline CAGTGAGGACGGAAGT vs & ACTTCCGTCCTCACTG & 62.2 \\
\hline GTCGTAGCATCGCACC vs & GGTGCGATGCTACGAC & 64.8 \\
\hline CAACCACGCCTGTCCA vs & TGGACAGGCGTGGTTG & 65.9 \\
\hline AAAGGAACTCTCCGCG vS & CGCGGAGAGTTCCTTT & 63.5 \\
\hline GACAAAGCCGACACGT vs & ACGTGTCGGCTTTGTC & 64.5 \\
\hline GCCTCTGTGTCGCATC vs & GATGCGACACAGAGGC & 64.8 \\
\hline GCTCGAGC VS & GCTCGAGC & 43.7 \\
\hline CTCGCGAG VS & CTCGCGAG & 43.2 \\
\hline
\end{tabular}




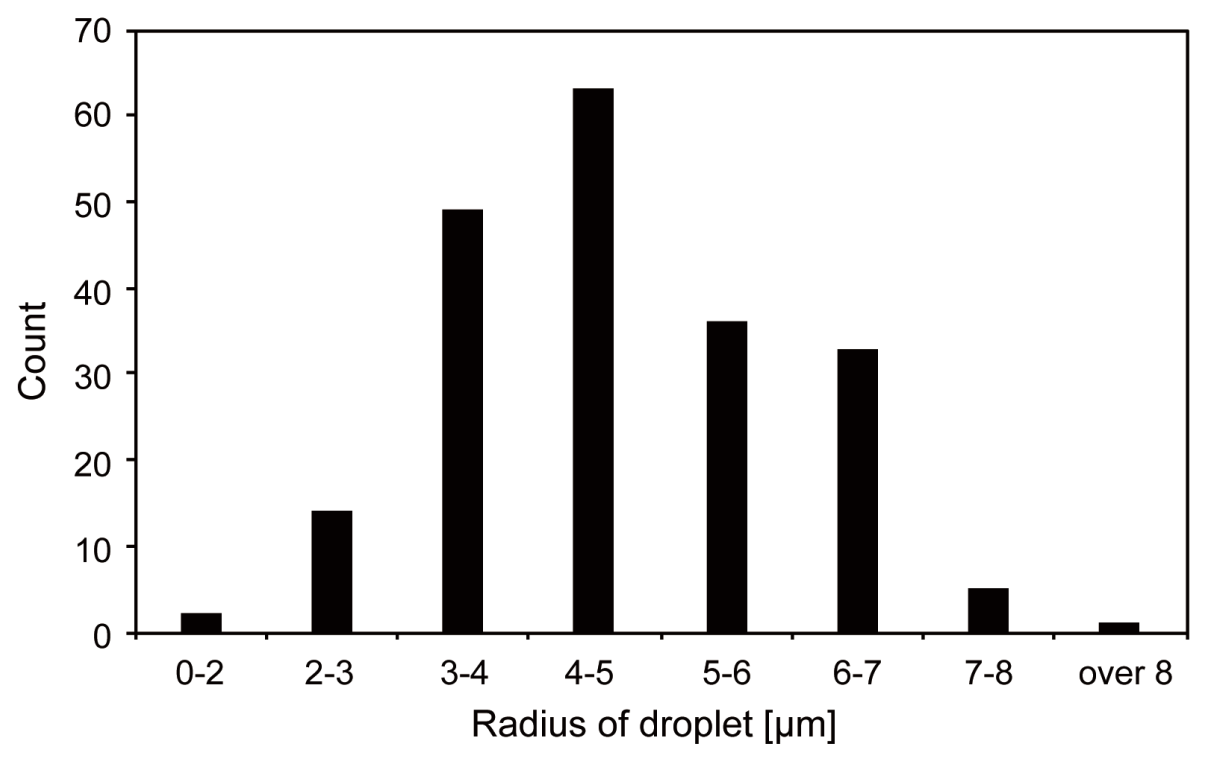

Figure S1: Size distribution of generated droplets. The generated droplet radius was $4.7 \pm 1.3 \mu \mathrm{m}$ (mean \pm standard deviation). The number of measured droplets was 203. 
(a)
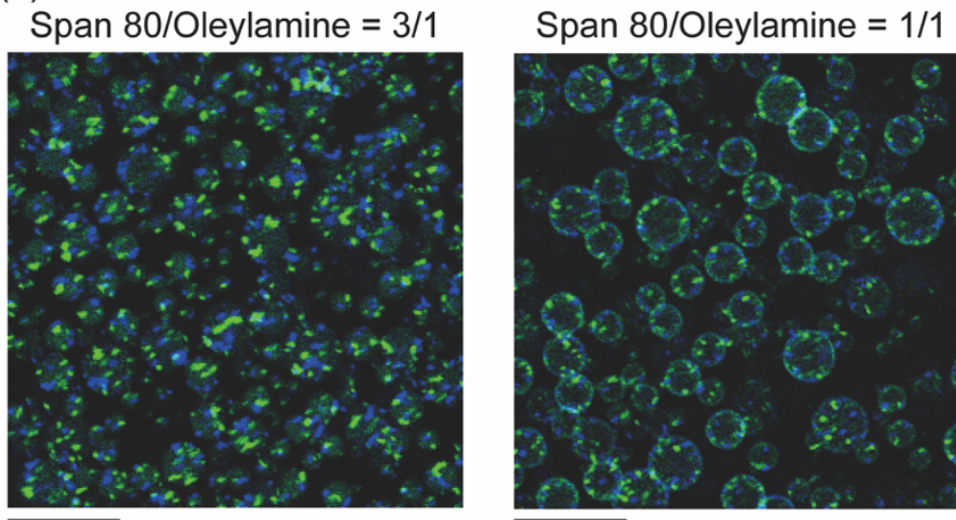

Span 80/Oleylamine $=1 / 3$
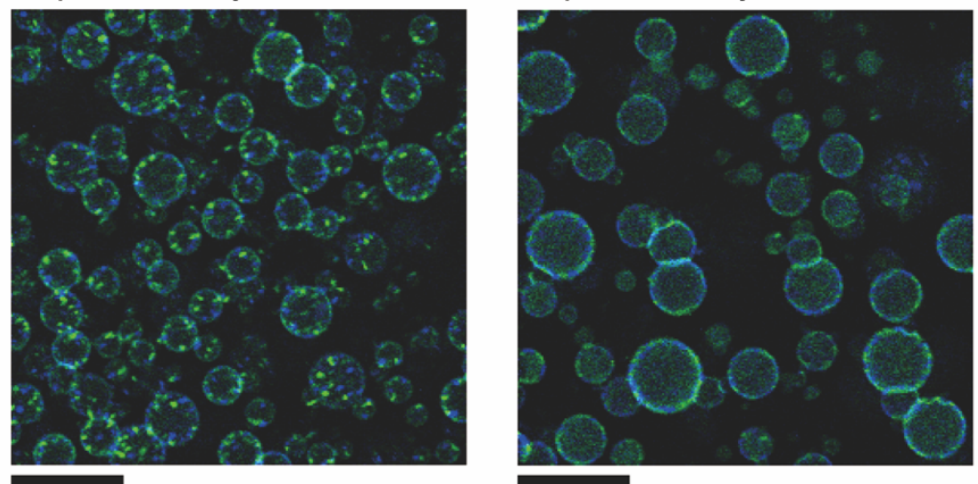

(b)
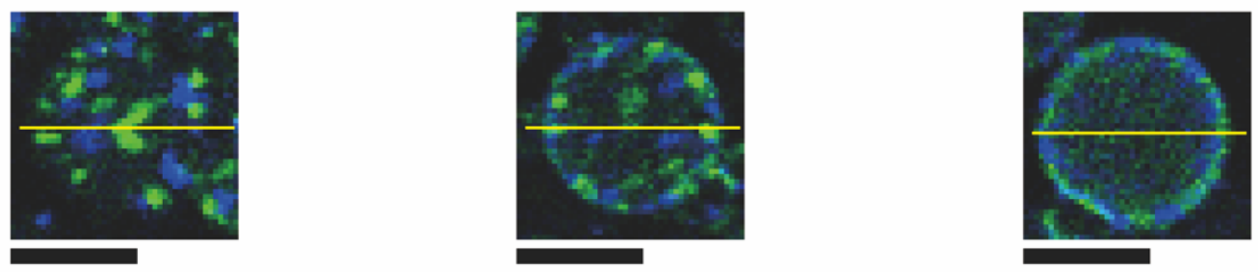

(c)
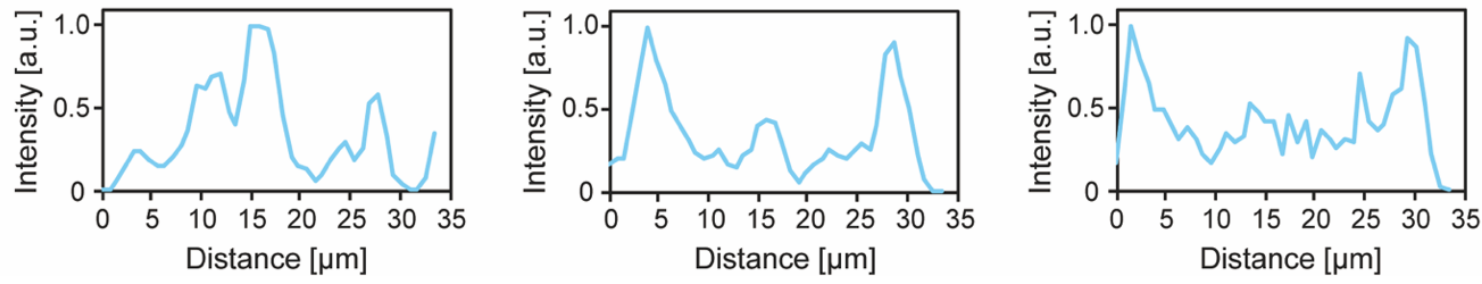

Figure S2: Effects of the Span 80/oleylamine ratio in the adsorption of DNA nanostructures onto the droplet interface. Note that the $\mathrm{Y}$ - and ${ }^{\text {orth }} \mathrm{Y}$-motif concentrations were $5 \mu \mathrm{M}$ each. Representative confocal microscopy images using Span 80/oleylamin at the ratios of $3: 1,1: 1$, and $1: 3$ (a) magnified images (b) (from left to right). At the Span 80 /oleylamine ratios of $3: 1$ and 1:1, DNA hydrogel particles were formed inside the droplets, indicating that DNA nanostructures were not fully adsorbed onto the interface. Scale bars: $50 \mu \mathrm{m}$ in (a) and $20 \mu \mathrm{m}$ in (b). (c) Fluorescence intensity profiles on the yellow lines shown in (b). 


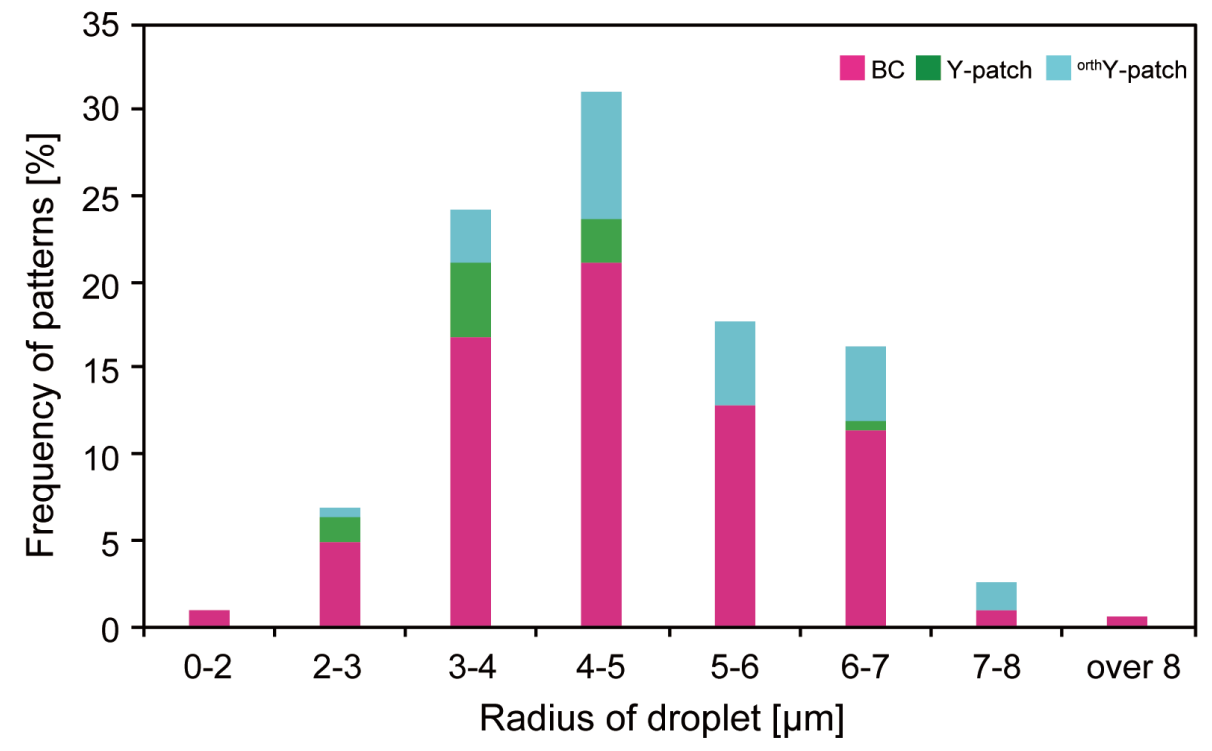

Figure S3: Frequency of each pattern in different droplet sizes. Size dependency on pattern frequency was not confirmed. The number of analyzed droplets was 203. 

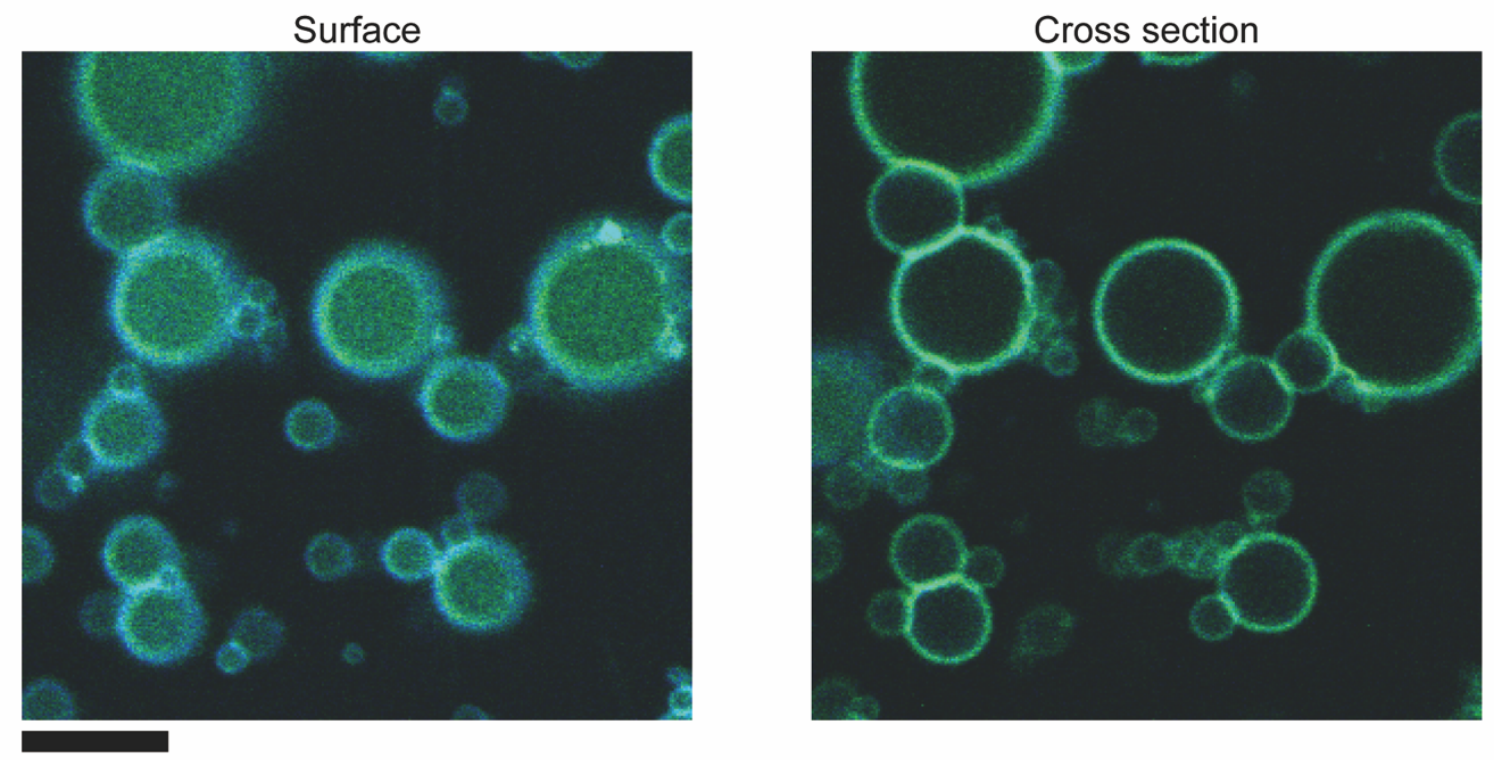

Figure S4: Confocal microscopy images of the droplets containing $\mathrm{Y}$ - and ${ }^{\text {orth }} \mathrm{Y}$-motifs without sticky ends, each at a concentration of $2.5 \mu \mathrm{M}$. No patterns were observed, suggesting that the formed patterns were due to the orthogonality of sticky end sequences in the motifs. Scale bar: $20 \mu \mathrm{m}$. 


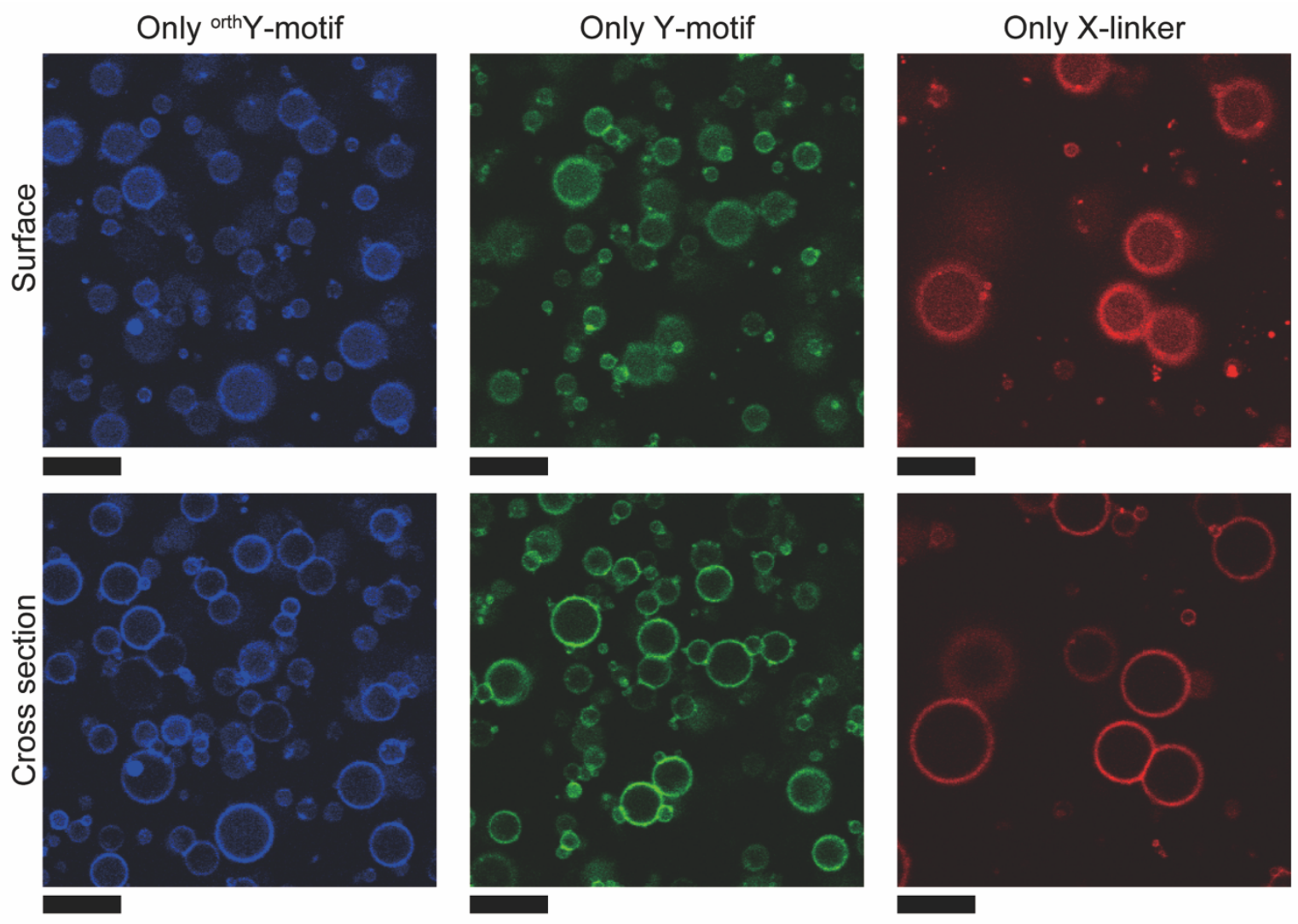

Figure S5: Confocal microscopy images of the droplets containing only ${ }^{\text {orth }} \mathrm{Y}-, \mathrm{Y}-\mathrm{or} \mathrm{X}$ linkers, each at a concentration of $5 \mu \mathrm{M}$. Scale bars: $20 \mu \mathrm{m}$. 


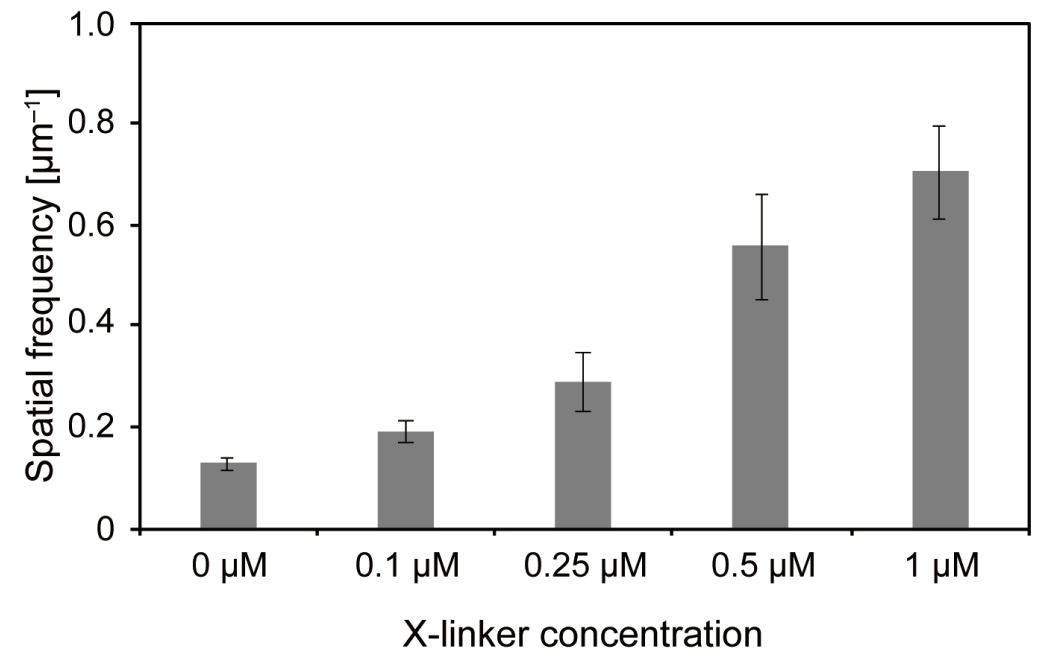

Figure S6: Size evaluation of the ${ }^{\text {orth }} Y$-motif region on the droplet interface analyzed using Fourier transformation. The data show the mean \pm standard error. 

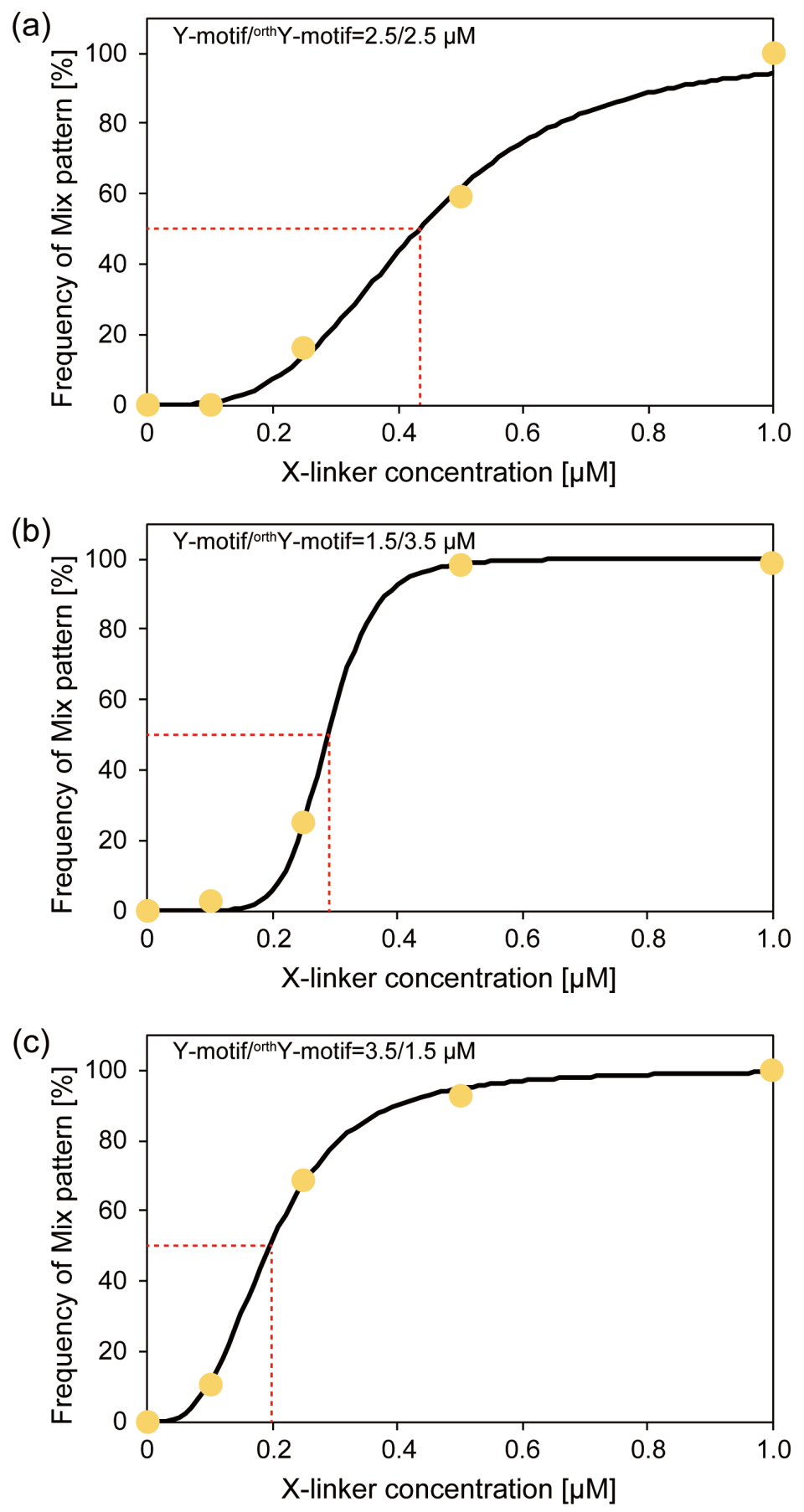

Figure S7: Fitting of appearance frequency of the Mix pattern to a Hill-type sigmoidal

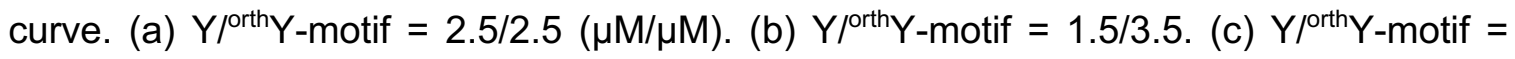
3.5/1.5. Yellow dots and black solid lines represent experimental results and fitting curves, respectively. Red dashed lines show the X-linker concentrations resulting in a $50 \%$ Mix pattern, which were $0.43 \mu \mathrm{M}$ in (a), $0.29 \mu \mathrm{M}$ in (b), and $0.20 \mu \mathrm{M}$ in (c). 
(a)

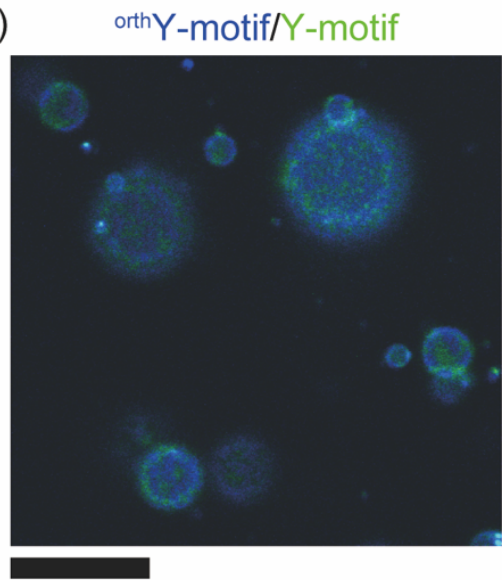

(b)

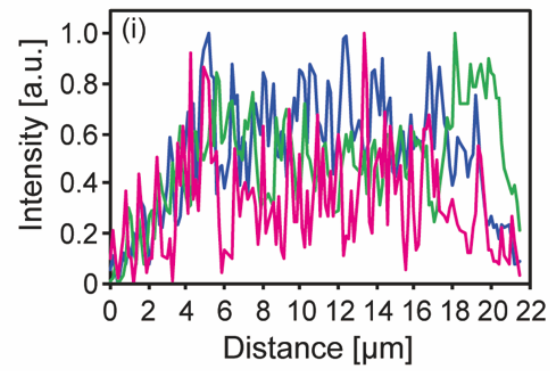

X-linker
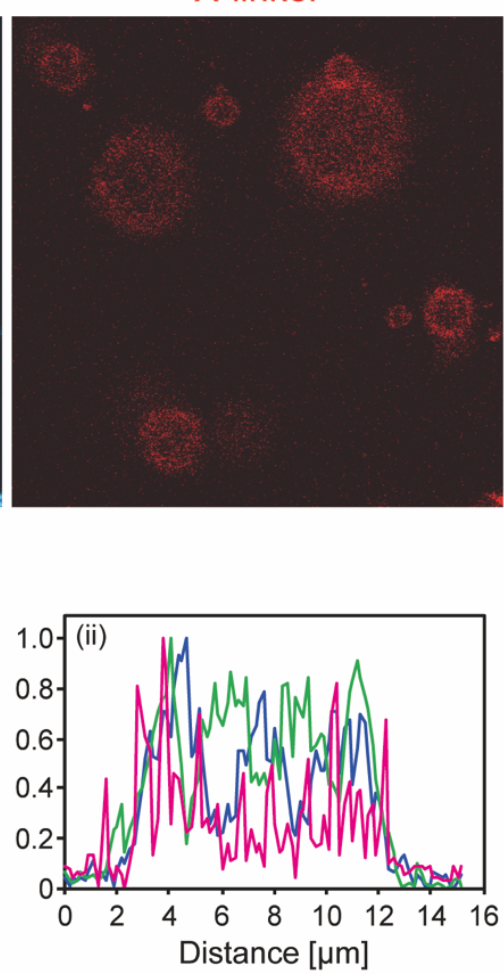

Marge
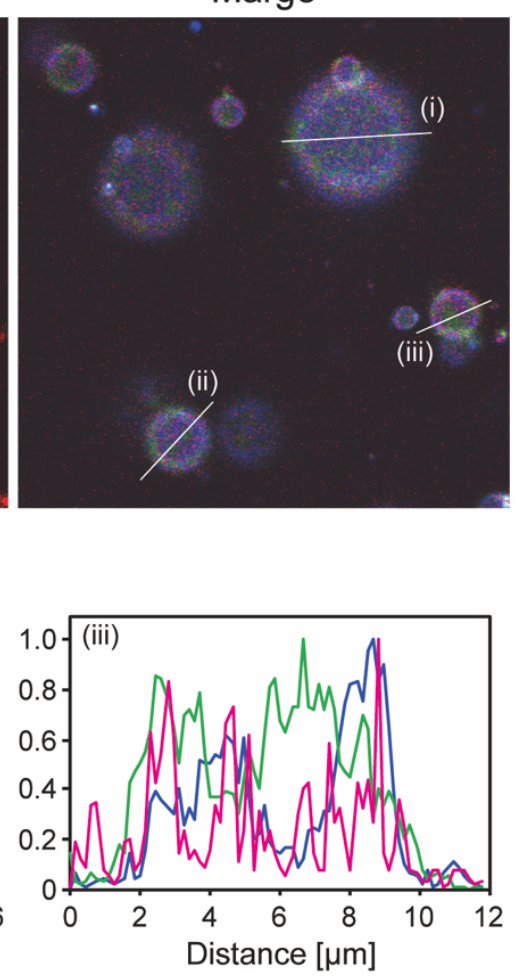

Figure S8: X-linker distribution at $\mathrm{Y}$-/orth $\mathrm{Y}$-/X-linker= 2.5/2.5/0.25 $\mu \mathrm{M}$. (a) Microscopy images of the droplet surfaces. Scale bar: $20 \mu \mathrm{m}$. (b) Normalized intensity profiled at the lines shown in the right images in (a). (i), (ii), and (iii) correspond to the white lines in the image. The blue, green, and magenta colors represent the $\mathrm{Y}-$, ${ }^{\text {orth }} \mathrm{Y}-$, and the $\mathrm{X}$-linkers, respectively. 
(a) Y-motif/orthY-motif $=3.5 / 1.5[\mu \mathrm{M}]$
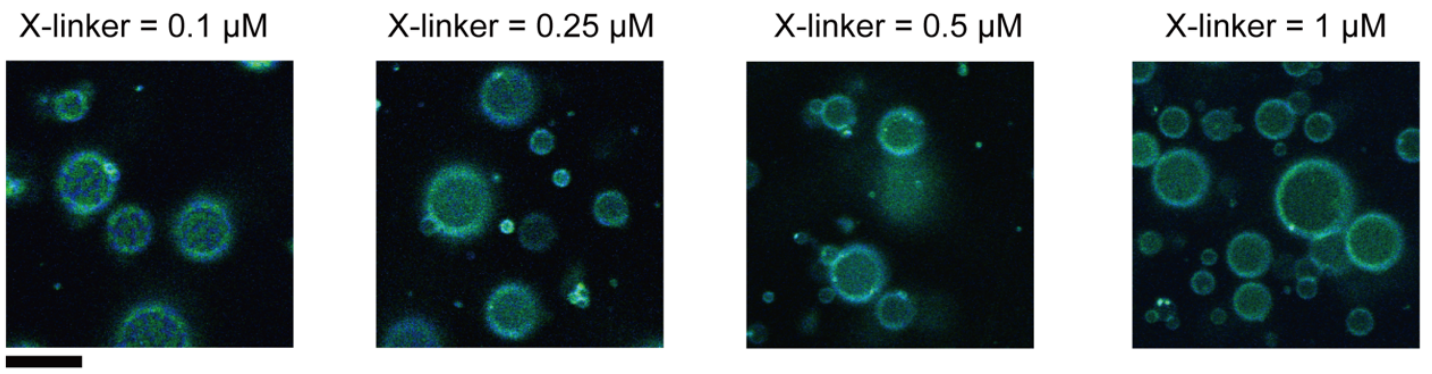

(b) Y-motif/orthY-motif $=1.5 / 3.5[\mu \mathrm{M}]$
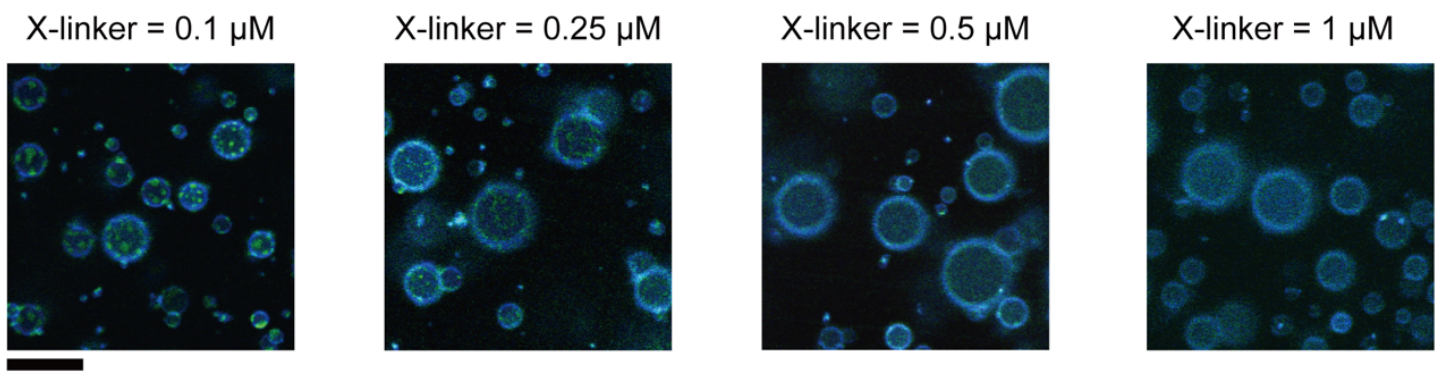

Figure S9: Microscopy images of the droplet surfaces under imbalanced $Y$-/orth $Y$-motif concentration with different $X$-linker concentration. (a) 3.5/1.5 $\mu \mathrm{M}$ of $\mathrm{Y} /$ orth $^{\text {- }}$-motifs with 0.1 , $0.25,0.5$, and $1 \mu \mathrm{M} X$-linkers. (b) $1.5 / 3.5 \mu \mathrm{M}$ of $\mathrm{Y}^{\text {orth }} \mathrm{Y}$-motifs with $0.1,0.25,0.5$, and $1 \mu \mathrm{M}$ X-linkers. Scale bars: $20 \mu \mathrm{m}$. 

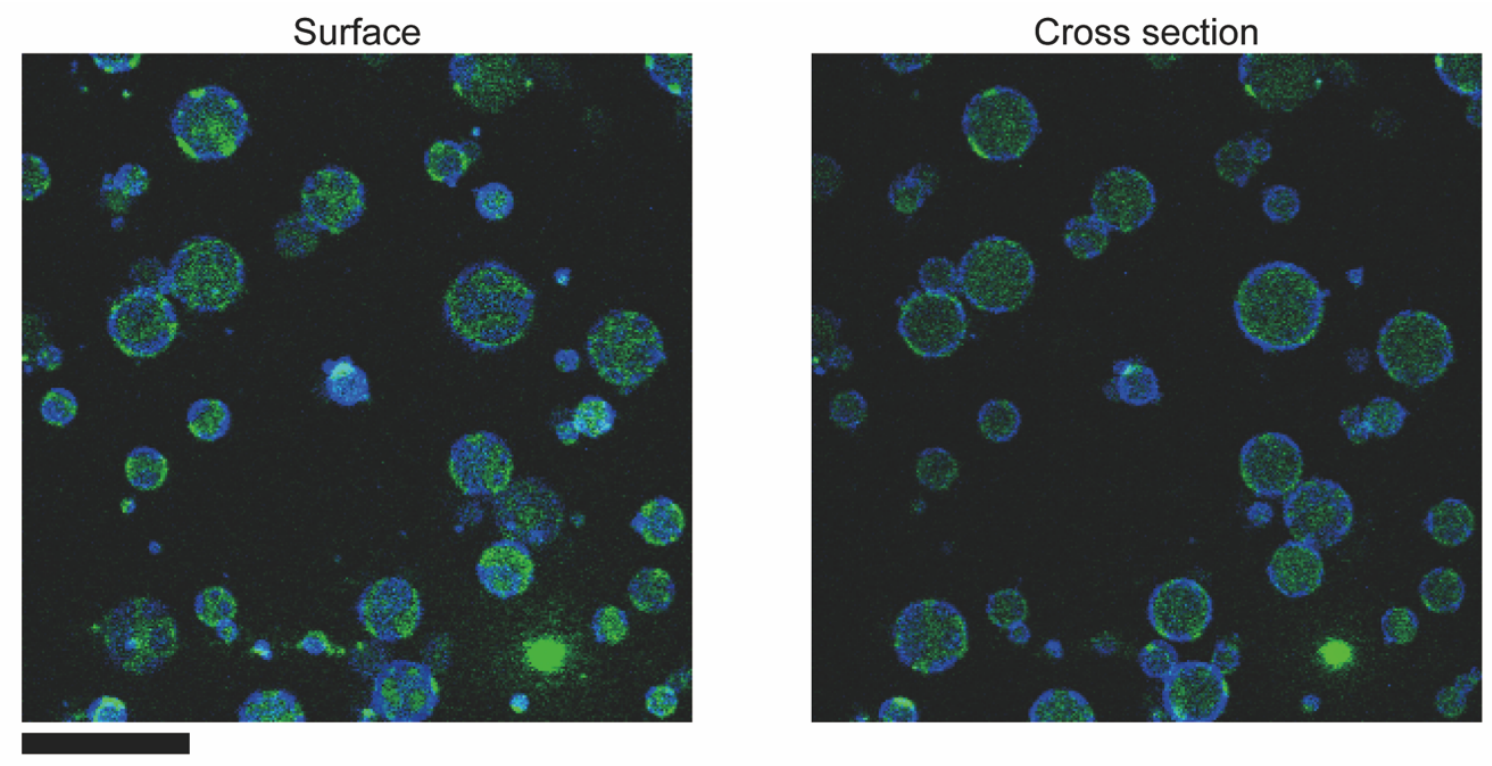

Figure S10: Microscopy images of phase-separated hydrogels formed on droplet surfaces that were generated using an equimolar mixture of DOPC and DOTAP. Scale bar: $30 \mu \mathrm{m}$.

\section{SI References}

1. A. V. HILL, The possible effects of the aggregation of the molecules of haemoglobin on its dissociation curves. J. Physiol. 40, iv-vii (1910).

2. N. R. Markham, M. Zuker, DINAMelt web server for nucleic acid melting prediction. Nucleic Acids Res. 33, W577-581 (2005). 